\title{
Metabolome and transcriptome analysis reveals the molecular profiles underlying the ginseng response to rusty root symptoms
}

Xingbo Bian ${ }^{1,2}$, Yan Zhao ${ }^{1}$, Shengyuan Xiao ${ }^{1,2^{*}}$, He Yang ${ }^{1,2}$, Yongzhong $\operatorname{Han}^{3}$ and Lianxue Zhang ${ }^{1,2^{*}}$

\begin{abstract}
Background: Ginseng rusty root symptoms (GRS) is one of the primary diseases of ginseng. This disease leads to a severe decline in the quality of ginseng. It has been shown that the occurrence of GRS is associated with soil environmental degradation, which may involve changes in soil microbiology and physicochemical properties.

Results: In this study, GRS and healthy ginseng $(\mathrm{HG})$ samples were used as experimental materials for comparative analysis of transcriptome and metabolome. Compared with those in HG samples, 949 metabolites and 9451 genes were significantly changed at the metabolic and transcriptional levels in diseased samples. The diseased tissues' metabolic patterns changed, and the accumulation of various organic acids, alkaloids, alcohols and phenols in diseased tissues increased significantly. There were significant differences in the expression of genes involved in plant hormone signal transduction, phenylpropanoid biosynthesis, the peroxidase pathway, and the plant-pathogen interaction pathway.

Conclusion: The current study involved a comparative metabolome and transcriptome analysis of GRS and HG samples. Based on the findings at the transcriptional and metabolic levels, a mechanism model of the ginseng response to GRS was established. Our results provide new insights into ginseng's response to GRS, which will reveal the potential molecular mechanisms of this disease in ginseng.
\end{abstract}

Keywords: Panax ginseng, Rusty root, Metabolome, Transcriptome, Environmental stress

\section{Background}

Ginseng (Panax ginseng Mayer) is one of the most important foods and herbs because of its high nutritional value and medicinal potential. As a perennial plant, the active ingredients in ginseng accumulate over time [1]. Ginseng has higher requirements for the growing environment and a longer growing period, making it susceptible to various diseases during growth, thus affecting its yield and quality.

\footnotetext{
* Correspondence: xia003435@163.com; zlx864@163.com

${ }^{1}$ College of Chinese Medicinal Materials, Jilin Agricultural University, Changchun 130118, Jilin Province, China

Full list of author information is available at the end of the article
}

Ginseng rusty root symptoms (GRS) is one of the most common diseases in ginseng cultivation and production. It produces reddish-brown spots on the periderm of ginseng roots, and over time, the spots may gradually expand, leading to a decline in the commodity grade and quality of ginseng. Whether GRS is an infectious disease or a non-infectious physiological disease is still controversial, and some scholars in China have tried to answer this question [2,3]. Previous research on American ginseng (Panax quinquefolius L.) showed that rusty roots might be a defence mechanism against the invasion of certain fungi, resulting in the stimulation of phenolic

C C The Author(s). 2021 Open Access This article is licensed under a Creative Commons Attribution 4.0 International License, which permits use, sharing, adaptation, distribution and reproduction in any medium or format, as long as you give appropriate credit to the original author(s) and the source, provide a link to the Creative Commons licence, and indicate if changes were made. The images or other third party material in this article are included in the article's Creative Commons licence, unless indicated otherwise in a credit line to the material. If material is not included in the article's Creative Commons licence and your intended use is not permitted by statutory regulation or exceeds the permitted use, you will need to obtain permission directly from the copyright holder. To view a copy of this licence, visit http://creativecommons.org/licenses/by/4.0/ The Creative Commons Public Domain Dedication waiver (http://creativecommons.org/publicdomain/zero/1.0/) applies to the data made available in this article, unless otherwise stated in a credit line to the data. 
compound production $[4,5]$. Some research results have indicated that fungal infection is the cause of GRS [6-8]. However, this disease's effect on ginseng at the transcriptional and metabolic levels and the response mechanism of ginseng are still unclear.

RNA-seq is a method of transcriptome analysis using deep sequencing technology [9]. This method can detect almost all genes and pathways related to a physiological response with high sensitivity [10]. In recent years, increasing studies have used the Illumina RNA-eq platform based on transcriptome analysis to explore plant responses to abiotic or biological stresses and understand their associated molecular mechanisms [11-14]. Metabolomics, similar to transcriptomics, is an essential part of systems biology. Metabolomics aims to detect and quantify all metabolites in biological samples [15]. This method is gradually being applied in medicinal plant research and plant pathology as an essential bridge connecting genes, metabolites, and phenotypes. In the research field of plant pathology, this method has been gradually combined with other research methods and applied to research on plant disease diagnosis [16], plant disease resistance [17], plant noninfectious diseases [18, 19], and other fields showing good application prospects.

In this study, GRS and HG tissues from the same ginseng farm were selected as experimental materials. Transcriptome and metabolome analyses of GRS and HG tissues were performed using RNA-seq and ultra-high- performance liquid chromatography-tandem mass spectrometry (UHPLC-MS/MS) to elucidate the mechanism of the ginseng response to this disease. These findings provide not only valuable information for the prevention and treatment of GRS but also important insights for further understanding the molecular mechanism of the ginseng response to external stress.

\section{Results}

\section{Quality control of RNA-seq reads}

We performed RNA-seq analysis to compare the transcriptome between GRS and HG samples, and mainly analysed mRNA accumulation information obtained from the cDNA libraries. The sequencing of each ginseng sample produced over 100 million reads. After filtering out adaptors and low-quality reads, we obtained high-quality clean reads, accounting for more than $96 \%$ of the raw reads (Table S1).

\section{Differentially expressed genes (DEGs) of diseased tissues}

The DEGs between the GRS and HG samples are shown in the volcano plot and clustering map (Fig. 1a-b). The results showed that compared with the HG group, the expression of 4570 genes in the GRS group was upregulated, and that of 4881 genes was downregulated.

To find the gene functions of DEGs in ginseng rusty roots, we performed Gene Ontology (GO) and Kyoto Encyclopedia of Genes and Genomes (KEGG) pathway a

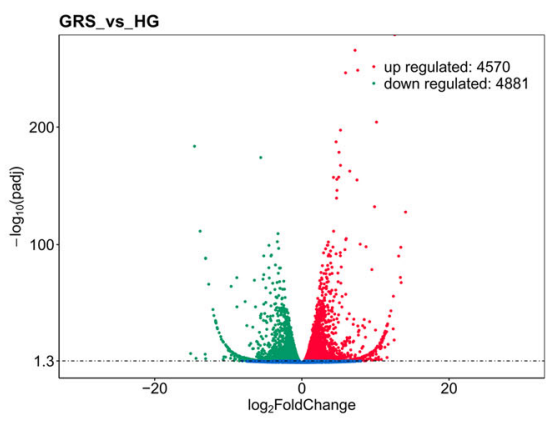

b

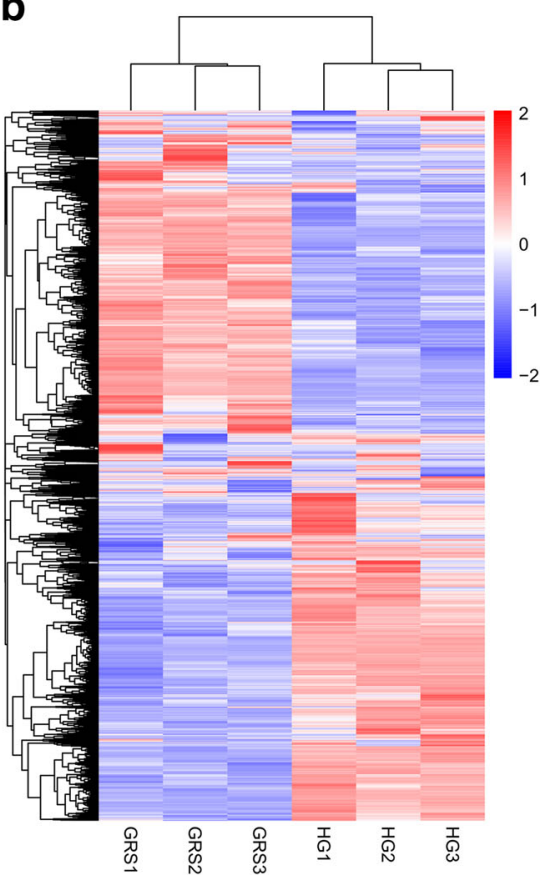

Fig. 1 Expression profiling changes of genes in diseased tissues. Volcano plot indicating upregulated and downregulated genes (a); heat map showing hierarchical clustering of DEGs (b). HG: healthy ginseng; GRS: ginseng rusty root symptoms 
analysis. GO is the international standard classification system for gene functions. All of the DEGs can be divided into three categories, including biological process (BP), cellular component (CC), and molecular function (MF).

GO enrichment histograms drawn after classification of the enriched GO terms are shown in Fig. 2a. Based on $\mathrm{GO}$ analysis of the transcriptionally upregulated mRNAs, the most significantly enriched BPs were "carbohydrate metabolic process," "steroid biosynthetic process," "oxidation-reduction process," "cell wall organization or biogenesis," and "response to oxidative stress," and the most significantly enriched CCs were "cell wall" and "beta-galactosidase complex." The most significantly enriched MFs were "hydrolase activity," "oxidoreductase activity," and "peroxidase activity." In addition, the histogram drawn according to transcriptionally downregulated mRNAs is shown in Fig. 2b. The results show that the most significantly enriched BPs were "oxidation-reduction process" and "terpenoid biosynthetic process," and the most significantly enriched CCs were "apoplast" and "cell wall." The most significantly enriched MFs were "iron ion binding," "hydrolase activity," and "peroxidase activity." All of the enrichment results are shown in Tables S2 and S3.

For KEGG analysis of DEGs, the most significantly involved pathways were "metabolic pathways," "biosynthesis of secondary metabolites," and "phenylpropanoid biosynthesis" (Fig. 2c). All enriched KEGG pathways containing DEGs are given in Table S4.

Differentially accumulated metabolites of diseased tissues UHPLC-MS/MS determined the composition of metabolites in diseased and healthy tissues, and 1101 and 649 compounds were analysed in ginseng root tissues in the positive ion mode and negative ion mode, respectively (Tables S5-S6). The most abundant metabolites extracted included lipids and lipid-like molecules, organic acids and derivatives, alkaloids, and phenylpropanoids.

As shown in Fig. 3a-b for both the positive ion mode and negative ion mode, the metabolites for the two kinds of ginseng tissues were clustered together based on principal component analysis (PCA). This result suggests that there were significant differences in the metabolism of the two samples. We used the supervised partial least squares discriminant analysis (PLS-DA) method to separate the samples based on critical metabolite information. The PLS-DA score plot showed significant separation between the two groups of samples (Fig. 3cd). Additionally, to evaluate the models' reliability, we performed the permutation test with 200 iterations. The cross-validation results showed that neither the positive ion model nor the negative ion model was overfitted
$\left(R^{2}=0.79\right.$ and $Q^{2}=-0.90, R^{2}=0.74$ and $Q^{2}=-0.82$, respectively) (Fig. 3e-f).

We extracted VIP values from the variable importance plots of the PLS-DA models to search for differentially accumulated metabolites in diseased ginseng tissues. Differentially accumulated metabolites were screened based on three criterions: VIP $>1, P<0.05$ and fold change $>2$. According to the above criteria, 595 and 344 metabolites were selected from the positive ion mode and negative ion mode, respectively. To better understand the chemical differences between diseased and healthy tissues, we plotted the heatmaps of 12 samples in positive and negative ion modes (Fig. 4a-b). Most of the differentially accumulated metabolites screened in positive ion mode had higher accumulation in diseased tissues (419 upregulated and 176 downregulated). The same phenomenon was observed in negative ion mode (285 upregulated and 59 downregulated). By matching these metabolites with those in the database, we finally identified 669 differentially accumulated metabolites, of which 508 were upregulated and 159 were downregulated (Table 1).

The differentially accumulated metabolites in the two tissues were mapped to the KEGG database. These metabolites were mainly enriched in multiple alkaloid biosynthesis pathways, such as "Biosynthesis of alkaloids derived from histidine and purine" and "Biosynthesis of alkaloids derived from ornithine, lysine and nicotinic acid." In addition, the extremely significant differences in "Stilbenoid, diarylheptanoid and gingerol biosynthesis, " "Phenylpropanoid biosynthesis," "Biosynthesis of plant hormones," and "Purine metabolism" are also noteworthy (Fig. 4c). The abundances of differentially accumulated metabolites across 9 metabolic pathways, as well as the significantly enriched differentially accumulated metabolites in KEGG metabolic pathways and those with a compound number $\geq 5$ are shown in Fig. $4 d$.

\section{Response of ginseng to disease}

The results of the GO and KEGG enrichment analyses showed the response of GRS tissues. Numerous DEGs in GRS tissues were involved in plant hormone signal transduction, lignin synthesis, plant-pathogen interactions, and oxidative stress compared with those in HG tissues.

We focused on the pathway "plant hormone signal transduction," involving 135 DEGs (Fig. S1-S2). Almost all genes involved in ABA signal transduction, such as soluble ABA receptors PYL/RCAR, protein phosphatases type-2C (PP2Cs), and SNF1-related protein kinase (SnRK2.3, SnRK2.10), were upregulated in the GRS group (Fig. S1B). The JAR1 and coronatine insensitive 1 (COI1) genes involved in JA signalling were upregulated, and the jasmonate-zim domain (JAZ) gene was 


\section{a}

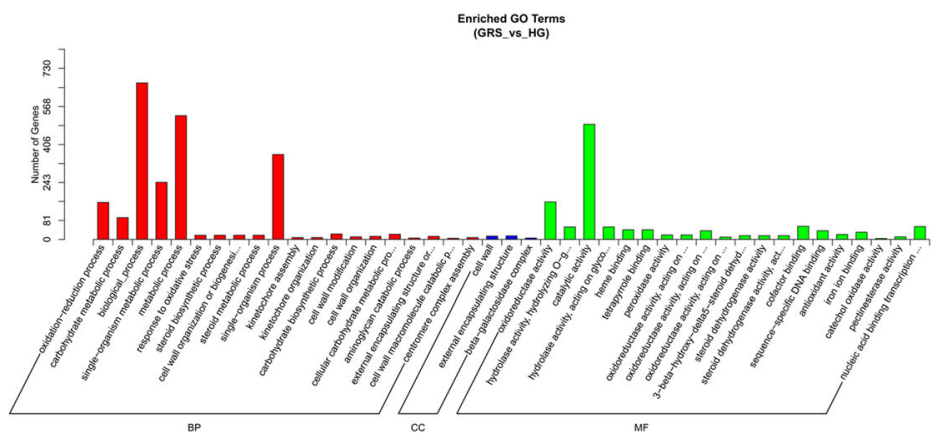

b

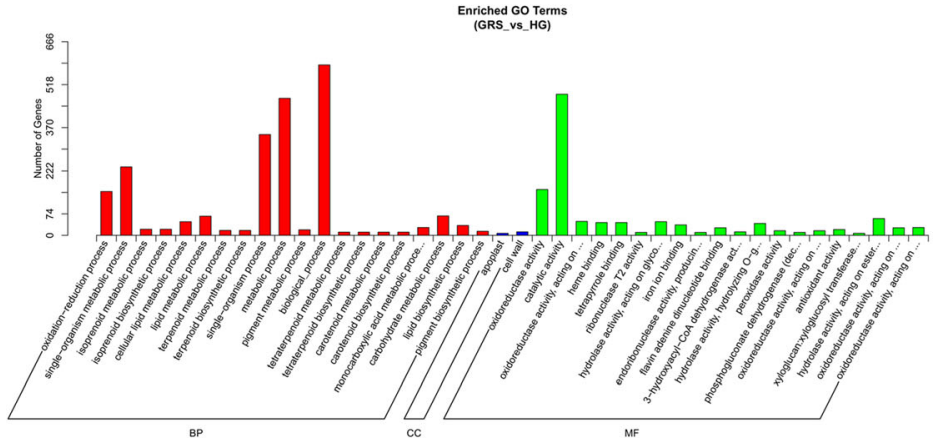

C

Statistics of Pathway Enrichment

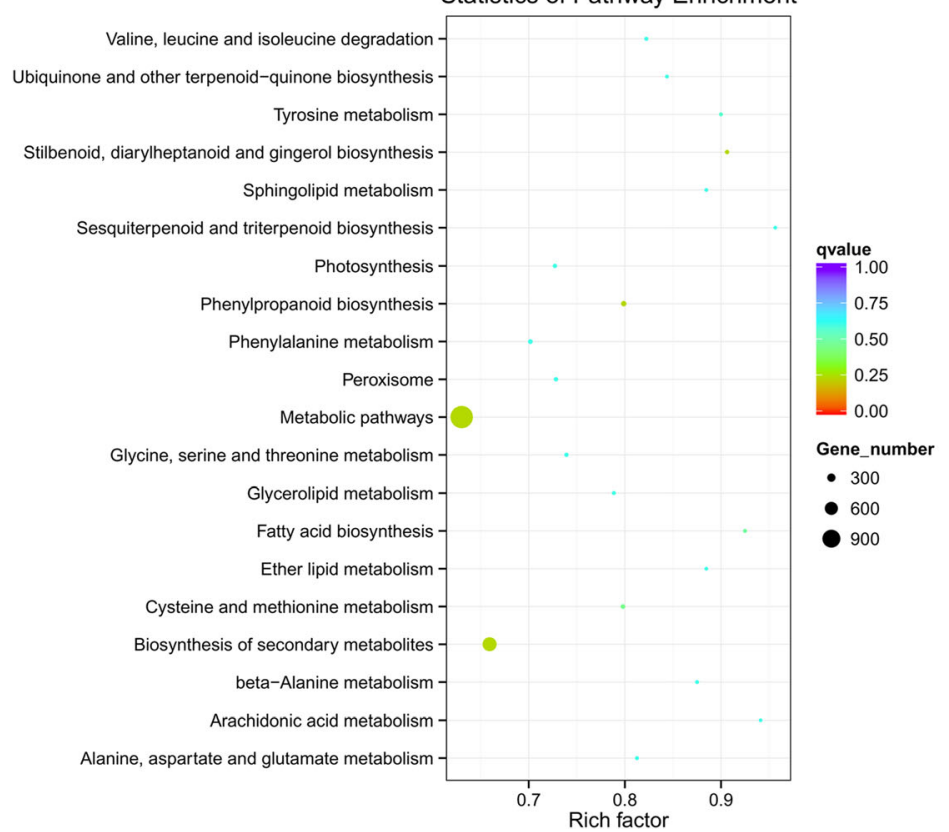

Fig. 2 Functional GO and KEGG pathway classification of DEGs in diseased tissues. a GO analysis of upregulated genes; b GO analysis of downregulated genes; c DEGs enriched the KEGG pathway scatterplot 
a

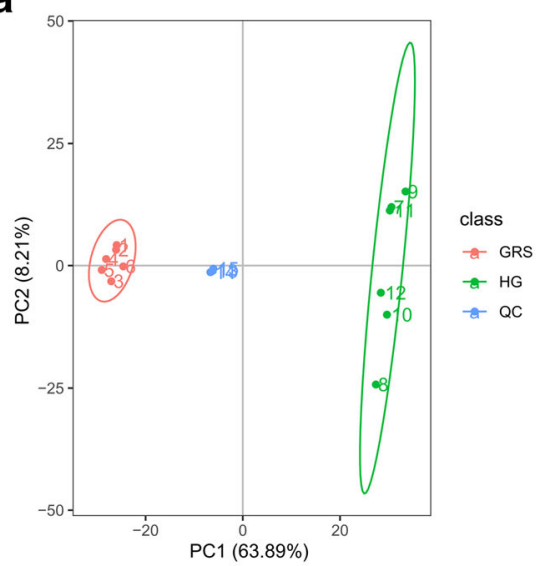

C

class - - GRS - - HG

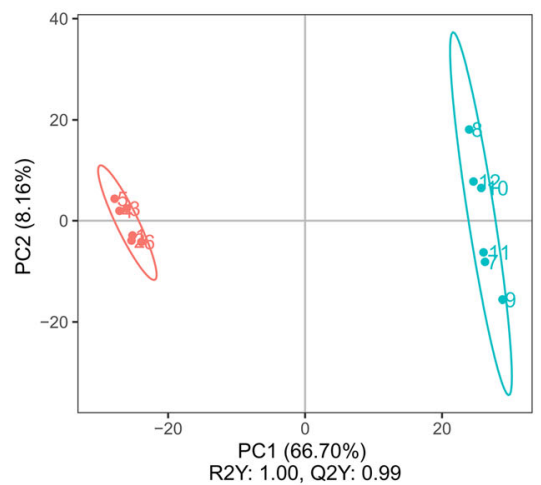

$$
\text { e }
$$

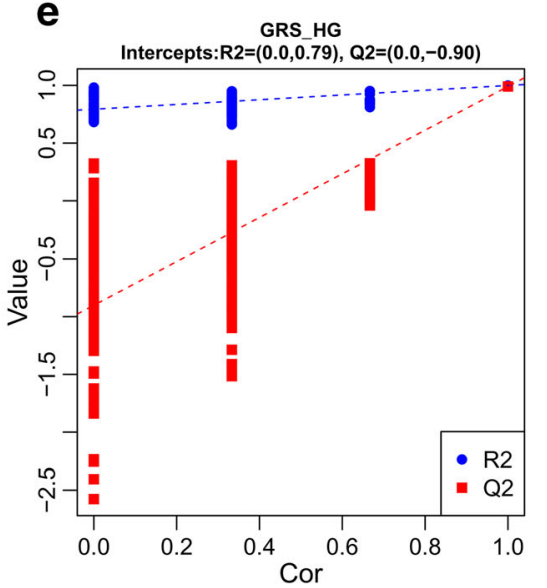

b

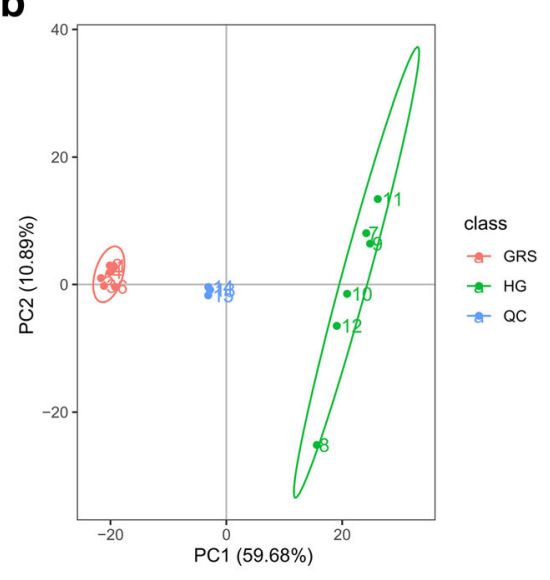

d

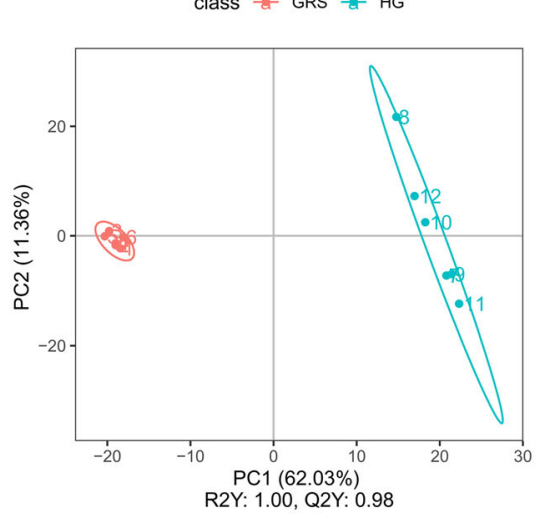

f

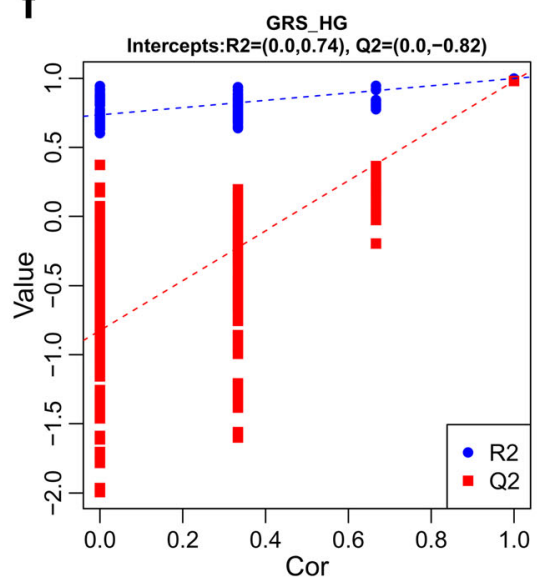

Fig. 3 Metabolomics profiling of diseased and healthy ginseng tissues. PCA plots of the positive ion mode (a) and negative ion mode (b); PLSDA plots of the positive ion mode (c) and negative ion mode (d); permutation tests for PLS-DA models in the positive ion mode (e) and negative ion mode (f). HG: healthy ginseng; GRS: ginseng rusty root symptoms

downregulated (Fig. S1C). Additionally, differential gene expression of mediator protein nonexpressor of pathogen-related gene 1 (NPR1), TGA factors, and pathogenesis-related gene PR-1 involved in SA signalling was found for GRS (Fig. S1D). The DEGs associated with plant hormone signal transduction are shown in Fig. S2. 

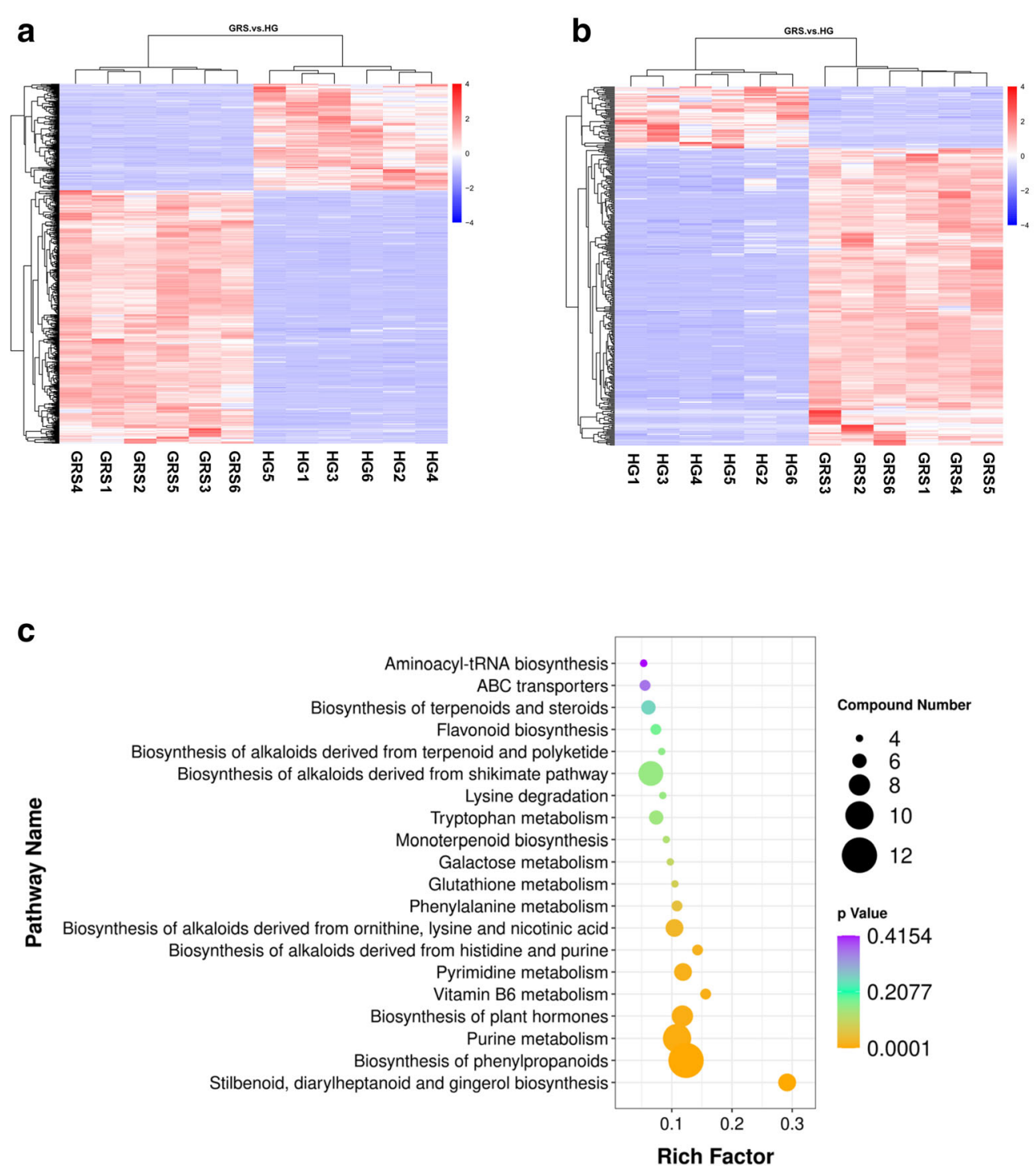

d
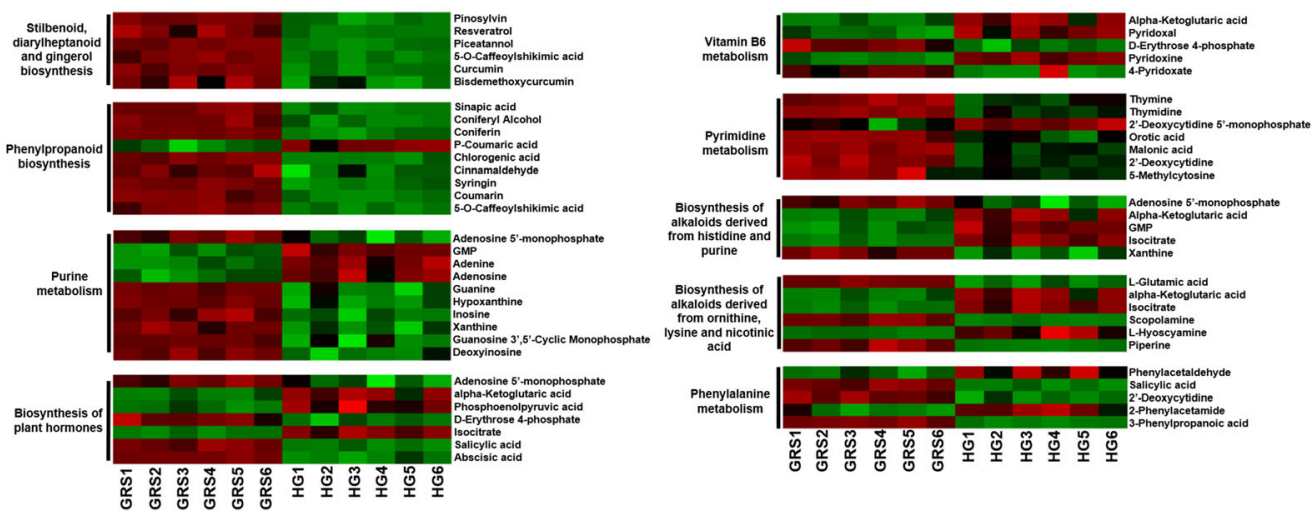

Fig. 4 (See legend on next page.) 
(See figure on previous page.)

Fig. 4 The hierarchical clustering analysis and heatmaps of differentially accumulated metabolites from diseased and healthy ginseng tissues identified in positive (a) and negative ion mode (b); differentially accumulated metabolites enriched the KEGG pathway scatterplot in the diseased tissues (c); changes in the accumulation of metabolites annotated to the KEGG metabolic pathway in ginseng rusty root symptoms tissues (d). HG: healthy ginseng; GRS: ginseng rusty root symptoms

In addition, we found that methyl jasmonate (MeJA), abscisic acid (ABA), and salicylic acid (SA) were strongly induced in diseased tissues (Fig. S1A).

There were 124 DEGs and 9 differentially accumulated metabolites involved in the "Phenylpropanoid biosynthesis" pathway (Table S4 and Fig. 4d). The detailed locations of differentially accumulated metabolites and DEGs in the pathway are shown in Fig. 5. In GRS tissues, enzymes closely related to lignin and phenol synthesis, such as phenylalanine ammonia-lyase (PAL), Omethyltransferase 1 (OMT1), 4-coumarate: coenzyme A ligase 1 (4CL1), and cinnamoyl coenzyme A reductase (CCR), were detected with a high expression level.

We analysed the "peroxisome" pathway, which is closely related to oxidative stress in plants. As shown in Fig. S3 and Fig. S4, we found that 59 DEGs were involved in the "peroxisome" pathway. Several PEX family genes, such as PEX14, PEX16, and PEX19, were downregulated in the GRS group. PXMP2 and MPV17 were involved in reactive oxygen species (ROS) metabolism and showed obvious downregulated expression. Additionally, catalase (CAT) and superoxide dismutase (SOD) in the antioxidant system were upregulated.

In our enrichment results, 96 DEGs were involved in the "plant-pathogen interaction" pathway (Table S4). The corresponding KEGG pathway is shown in Fig. S5. Several genes, including the respiratory burst oxidase homologue (RBOH) genes RBOHD and RBOHF, flagellin sensing 2 (FLS2), and WRKY family genes (WRKY33 and WRKY22), involved in pathogen-associated molecular pattern (PAMP)-triggered immunity (PTI) were differentially expressed. The RIN4, RPS2, and HSP genes related to effector-triggered immunity (ETI) were also differentially expressed. Furthermore, the defencerelated genes NHO1 and PR1 were upregulated (Fig. S6).

\section{Correlation analysis of differentially accumulated metabolites and DEGs}

We found that many DEGs and differentially accumulated metabolites were enriched in the same KEGG metabolic pathways in both types of tissue. These pathways include "Phenylpropanoid biosynthesis," "Phenylalanine metabolism," "Stilbenoid, diarylheptanoid and gingerol biosynthesis," and others (Fig. 6a). In addition, considering the role of the phenanthrene biosynthesis pathway in disease resistance and the stress response in plants, we constructed a correlation network graph of DEGs and differentially accumulated metabolites based on the Pearson correlation coefficient (PCC). As shown in Fig. 6b, a total of 36 DEGs and 8 differentially accumulated metabolites in the network were highly positively correlated $(\mathrm{PCC}>0.8)$. It is speculated that these genes and metabolites may play a key role in the response of ginseng to stress.

\section{Real-time quantitative polymerase chain reaction (qPCR) validation of mRNA expression}

To verify the accuracy of the RNA-seq results and provide the basis for further research, some genes from the four plant resistance pathways were selected for qRTPCR analysis. The expression level of these genes was demonstrated by qRT-PCR and transcriptome sequencing. The genes Pg_S1410.4 (UGT72E1), Pg_S1825.18 (CCR1), Pg_S2641.9 (CSD1), Pg_S7602.1 (MPV17), Pg S4621.10 (FLS2), Pg_S1378.3 (HSP90.1), Pg_S3064.15 (RP-1), and Pg_S0213.26 (JAZ) were analysed by qPCR (Fig. 7a). The expression levels of the corresponding

Table 1 Identification results of differential metabolites

\begin{tabular}{|c|c|c|c|c|c|c|c|c|c|}
\hline & $\begin{array}{l}\text { Organic acids and } \\
\text { derivatives }\end{array}$ & Alkaloids & Alcohols & Phenols & Phenylpropanoids & Terpene & Flavone & Lipids & Others \\
\hline up & 95 & 54 & 60 & 53 & 47 & 39 & 43 & 29 & 30 \\
\hline \multirow[t]{2}{*}{ down } & 36 & 32 & 10 & 4 & 7 & 13 & 7 & 15 & 6 \\
\hline & $\begin{array}{l}\text { Amino acids and } \\
\text { derivatives }\end{array}$ & Strides & Carbohydrates & Quinones & $\begin{array}{l}\text { Nucleotide and } \\
\text { derivatives }\end{array}$ & $\begin{array}{l}\text { Vitamins and } \\
\text { derivatives }\end{array}$ & $\begin{array}{l}\text { Indole } \\
\text { derivatives }\end{array}$ & $\begin{array}{l}\text { Plant } \\
\text { hormones }\end{array}$ & Amides \\
\hline up & 25 & 9 & 4 & 6 & 5 & 2 & 4 & 3 & 2 \\
\hline down & 8 & 8 & 5 & 1 & 2 & 3 & 0 & 0 & 0 \\
\hline
\end{tabular}




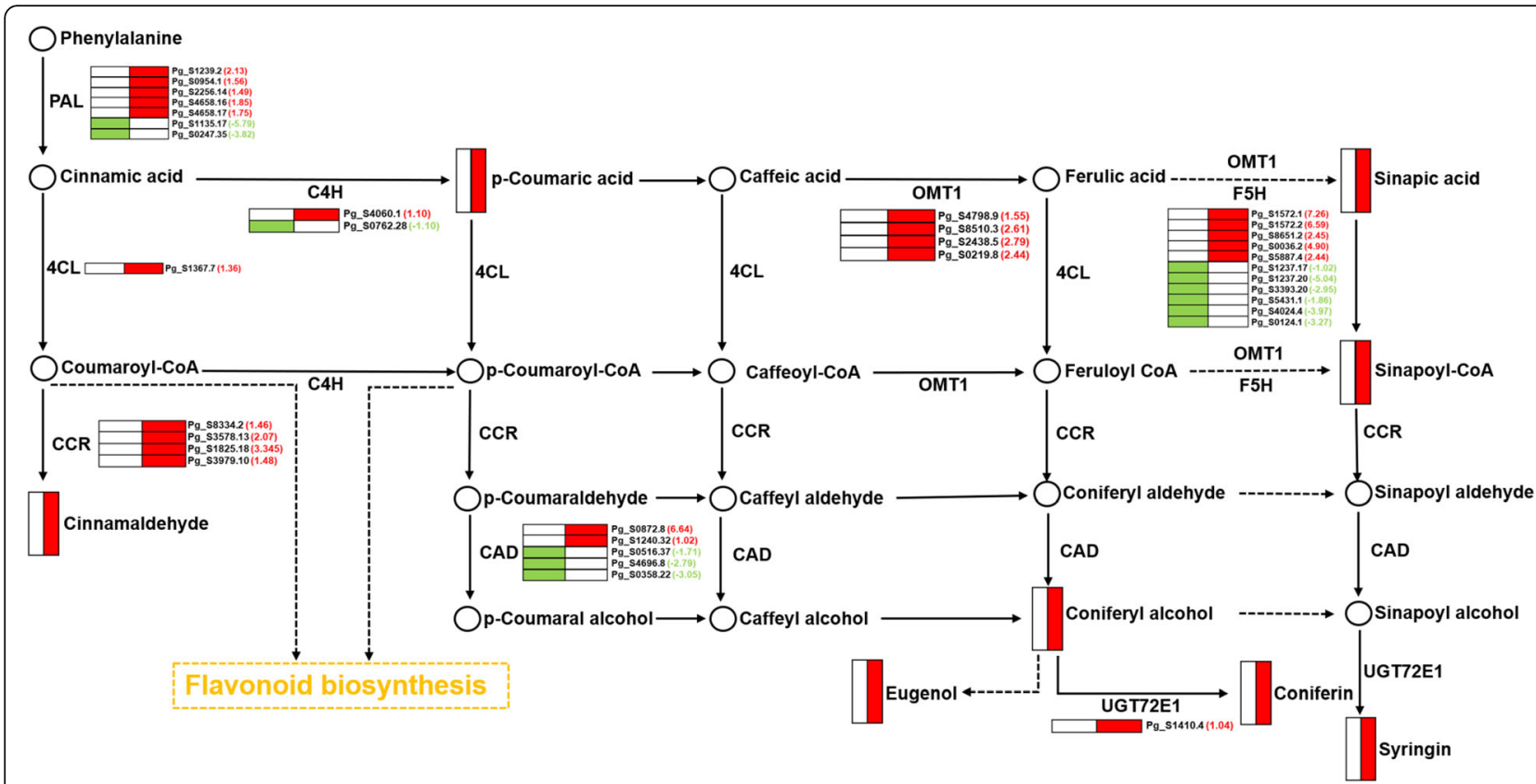

Fig. 5 DEGs and differentially accumulated metabolites related to phenylpropanoid biosynthesis. The different color indicates the DEGs and differentially metabolites in GRS compared with HG (Red frame: up-regulation; green frame: down-regulation). PAL, phenylalanine ammonia-lyase; C4H, cinnamate 4-hydroxylase; 4CL, 4-coumarate: CoA ligase; CCR, cinnamoyl-CoA reductase; CAD, cinnamyl alcohol dehydrogenase; OMT1, caffeic acid 3-Omethyltransferase 1; F5H, ferulate-5-hydroxylase

mRNAs obtained by RNA-seq are shown in Fig. 7b. All validation results fully proved the reliability and accuracy of the transcriptome sequencing data.

\section{Discussion}

Metabolites and total RNAs were extracted from the same variety of 5-year-old ginseng planted in the same ginseng farm (due to its higher rusty root index). The samples were prepared from healthy and diseased ginseng tissues. The purpose was to ensure that ginseng samples experienced the same field management and climate changes during the growth process [20]. We conducted UHPLC-MS/MS and RNA-seq analysis of GRS and HG tissues. The results showed that in the GRS sample, 939 metabolites were differentially accumulated, and 9451 genes were significantly differentially expressed. Based on previous studies and the results of this metabolomic and transcriptomic analysis, we propose a model to explain ginseng's response to GRS (Fig. 8).

\section{Hormone regulation in diseased tissues}

Plants produce a variety of hormones during growth. $\mathrm{ABA}, \mathrm{SA}$, and JA play an essential role in mediating plant defence responses to pathogens and abiotic stresses [21, 22]. At the transcriptional level, the ABA receptor PYR/PYL/RCAR, negative regulator $\mathrm{PP} 2 \mathrm{C}$, and positive regulator SnRK2 together comprise the regulatory system [23]. In GRS tissues, the genes that regulate
ABA signal transduction were differentially expressed, and $\mathrm{ABF}$ transcription factors were upregulated (Fig. S1B). Under JA-stimulated conditions, JA-Ile, synthesized by JAR1, binds to the receptor COI1, leading to the degradation of the repressor protein JAZ, which upregulates the expression level of JA target genes (Fig. $\mathrm{S} 1 \mathrm{C})[24,25]$. NPR1 is one of the key regulatory elements of SA-dependent PR gene activation and interacts with GTA factors to regulate PR gene expression [26, 27]. We found differential expression of genes that are by-products of NPR1 (BOP2 and NPR3) and multiple TGA transcription factors genes, which resulted in the upregulation of PR-1 gene expression (Fig. S1D). In addition, three plant hormones and hormone-related signalling molecules (ABA, SA, and MeJA) were detected by UHPLC-MS/MS, and all of them were significantly upregulated in diseased tissues (Fig. S1A). Therefore, the accumulation of hormones and the differential expression of genes related to hormone signal transduction in diseased tissues affected metabolic processes and eventually led to changes in growth patterns to withstand environmental stress.

\section{Immune system in diseased tissues}

Plants have two layers of defence against pathogens, PTI and ETI [28]. Here, compared to HG tissues, there were 96 DEGs associated with the plant-pathogen interaction pathway in GRS tissues. RBOH mediates ROS 

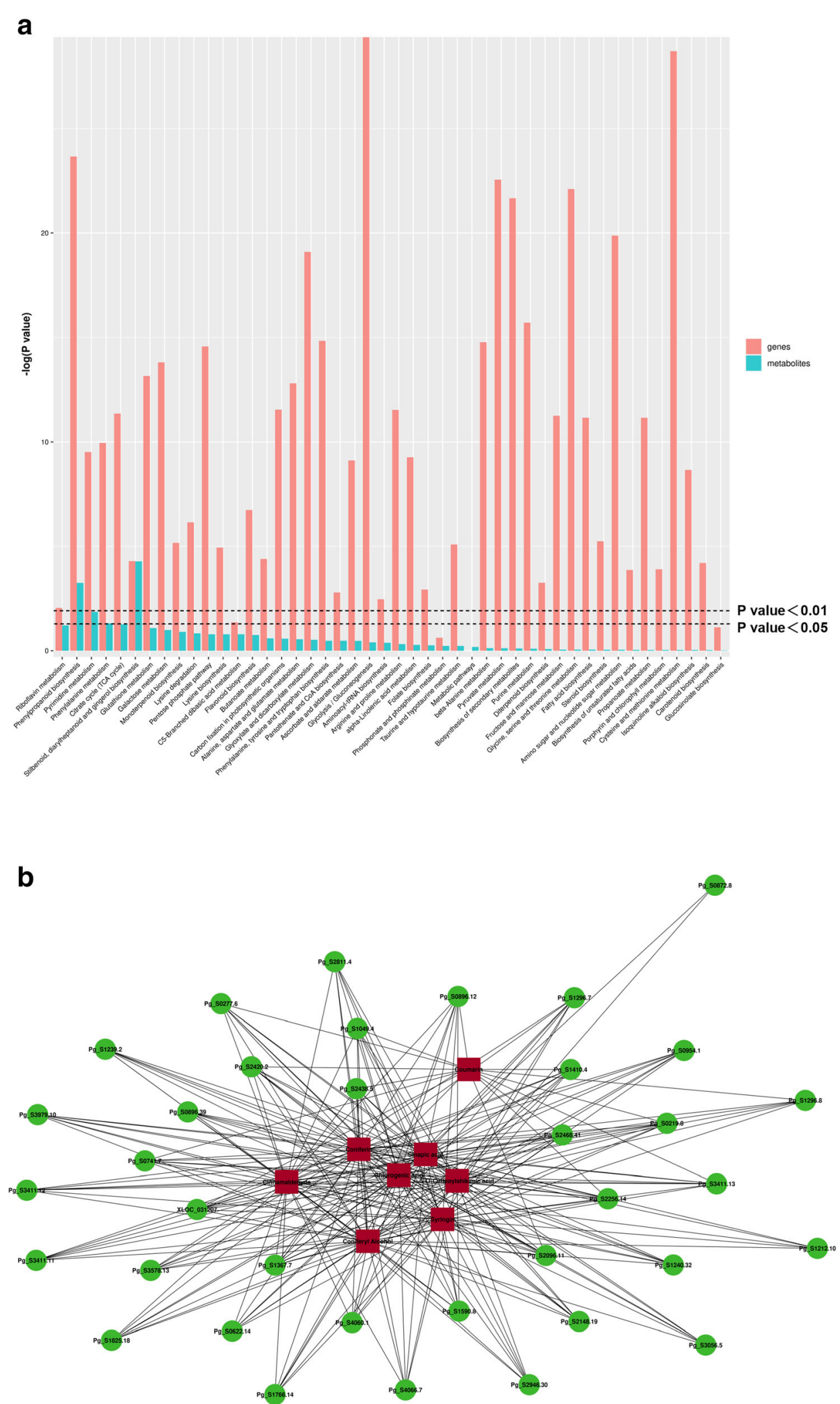

Fig. 6 a Statistical results of DEGs and metabolites enriched in the same KEGG pathway; $\mathbf{b}$ connection network between DEGs and differentially accumulated metabolites in phenylpropanoid biosynthesis pathway 


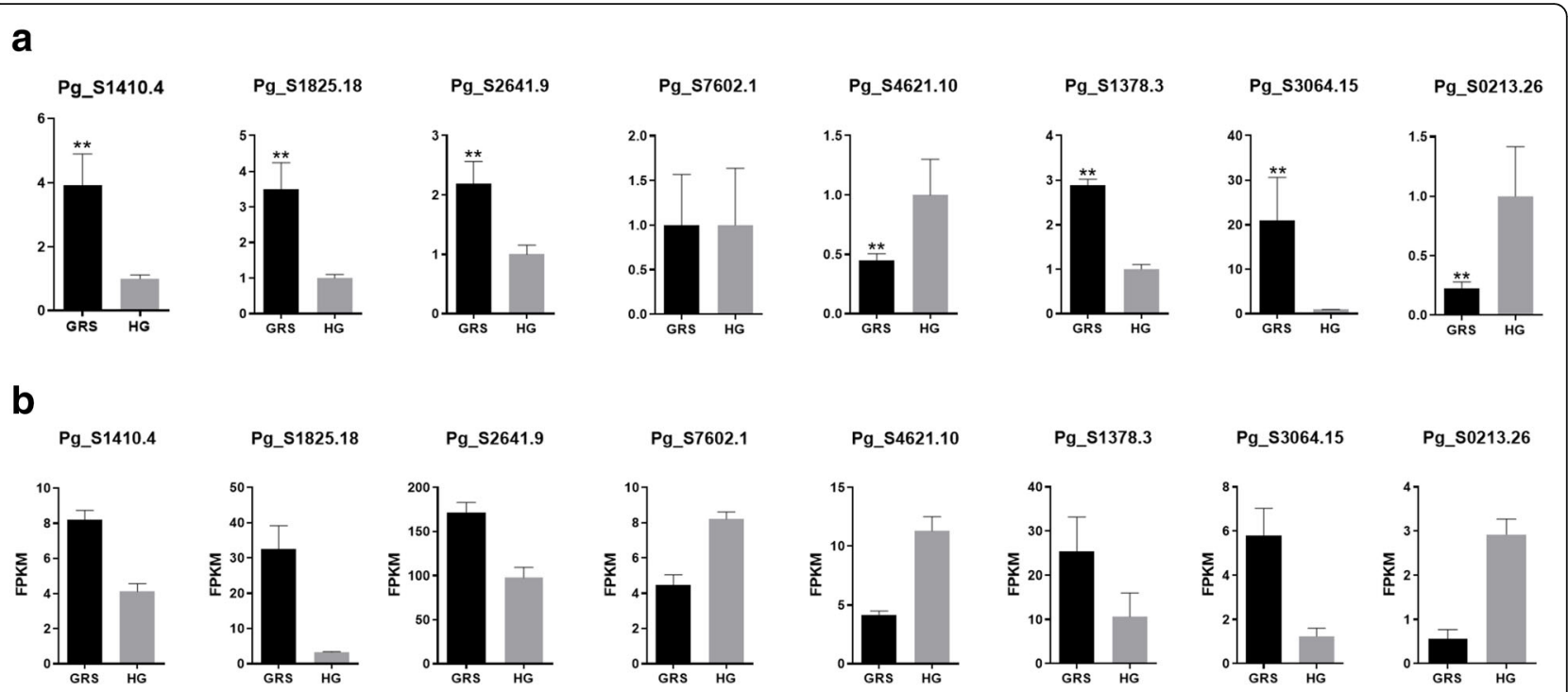

Fig. 7 qRT-PCR validation of significant DEGs. a qRT-PCR, the data were presented as the mean \pm SEM $(n=3) ;{ }^{* *} p<0.05$; b RNA-seq. HG: healthyginseng; GRS: ginseng rusty root symptoms

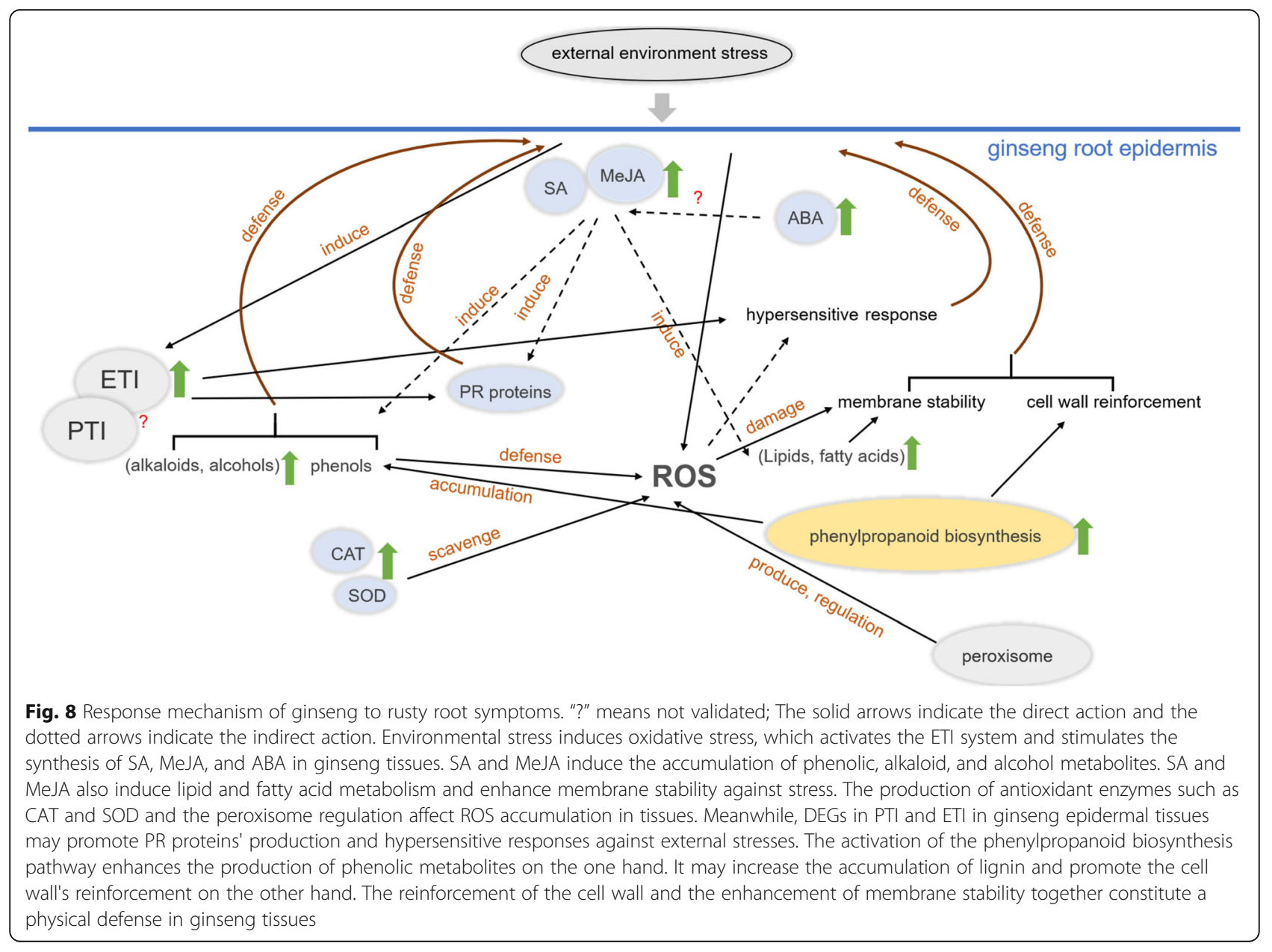


generation, which is strongly related to both PTI and ETI $[29,30]$. RBOHD and RBOHF are considered to be critical components of plant defences [31]. FLS2 is a pattern recognition receptor (PRR) that can trigger innate immunity in plants and is also involved in endocytosis [32]. WRKY33 plays a vital role as a transcription factor in plant resistance to pathogens [33]. The downregulation of the above genes, along with NOA1 and MEKK1, may indicate that part of PTI is repressed during GRS.

Interestingly, ETI seems to be activated in GRS samples. RPS2 is a resistance (R) protein in plants, while RIN4 is an essential negative regulator of natural plant immunity and has a regulatory effect on RPS2 [34]. HSP90.1 is required for RPS2 resistance and is rapidly induced in plants in the face of biological stress [35]. SGT1 positively regulates disease resistance produced by many $R$ proteins [36]. In our analysis, RIN4 was downregulated, and PRS2, SGT1, and HSP90.1 were upregulated. Furthermore, NHO1, an essential universal resistance gene in plants, was upregulated [37]. Overall, the immune system of diseased tissues was activated, which indirectly led to hypersensitivity and cell wall reinforcement.

\section{ROS-scavenging system in diseased tissues}

Exposure of plants to various biotic and abiotic stress conditions triggers rapid changes in reactive oxygen species (ROS) production and clearance [38]. ROS play an essential role in signalling pathways that regulate inflammatory and defence responses in plants, but their accumulation is often harmful to cells.

Peroxisomes are subcellular organelles with a basic oxidative type of metabolism and may be the primary intracellular ROS production site [39, 40]. KEGG enrichment analysis showed that both PXMP2 and MPV17, which are involved in ROS metabolism, were transcriptionally downregulated (Fig. S2). PXMP2 is a perovskite membrane protein with a channel-forming activity that allows free diffusion of compounds, such as $\mathrm{H}_{2} \mathrm{O}_{2}$ [41]. MPV17 is a protein involved in the production of ROS [40]. Several PEX family protein genes were differentially expressed, including PEX13 and PEX14, which are involved in matrix protein import, and PEX16 and PEX19, which are involved in membrane protein import [42, 43]. In addition, CAT and SOD genes were upregulated in diseased tissues. Thus, diseased tissues may respond to oxidative stress through peroxisomes and antioxidant systems [44].

The phenolic content is often considered to be closely related to plant tissues' total antioxidant capacity [45]. Studies have shown that flavonoids are also one of the main chemical defence substances in plants and can reduce various forms of reactive oxygen species in plant cells [46]. In our results, a significant upregulation of a large number of flavonoids and phenolics was found. Significant enrichment of the flavonoid biosynthetic pathway was also found by KEGG enrichment analysis (Fig. 4c). We also noted that multiple alcohol metabolites showed higher accumulation in diseased tissues. It was shown that the accumulation of alcohol usually plays a role in the fight against oxidative stress. Alcohols can act as intermediates of redox reactions with good scavenging capacity for free radicals and superoxide [47, 48]. It can be speculated that ginseng accumulates more alcohols in diseased tissues to cope with oxidative stress to improve its resistance. Overall, these compounds accumulate in diseased tissues of ginseng and may be jointly involved in the oxidative stress response.

\section{Physical and chemical defence in diseased tissues}

Lignin is a highly branched polymer of phenylpropanoid compounds and is the main component of the plant cell wall [49]. Lignin biosynthesis involves a complex genetic network containing many containing enzymes [50, 51]. Several key enzymes, including PAL, 4CL, CCR, OMT, and $C A D$, were upregulated $[52,53]$. In addition, phenols and flavonoids not only play a key role in the plant antioxidant process, but are also essential compounds involved in plant chemical defences [54]. Our analysis showed that both types of data (transcriptomic and metabolomic) were significantly enriched in the phenylpropanoid biosynthesis pathway (Fig. 6a). Considering that the phenylpropanoid biosynthesis pathway is closely related to phenolic production, flavonoid metabolism, and lignin formation, it is speculated that it may be a critical metabolic pathway in ginseng in response to GRS [55]. Therefore, we constructed a correlation network of differentially accumulated metabolites and DEGs in this metabolic pathway based on the correlation analysis results (Fig. 6b). The network includes several upstream regulatory genes of phenolic, lignin, and flavonoid synthesis, such as PAL (Pg_S1239.2, Pg_S0954.1, and Pg_S2256.14), 4CL (Pg_S1367.7), and C4H (Pg S4060.1), and precursor compounds, such as coniferin, sinapic acid, and coumarin. It is speculated that the upregulation of these genes and the accumulation of metabolites play a crucial role in the ginseng response to GRS.

Lipids are essential membrane components, and changes in their composition may help plants maintain cellular compartmentalization and membrane integrity [56]. In addition, free radicals are metabolized and produced when cells are under stress or in the process of injury. Therefore, reducing lipid peroxidation has an essential role in plant resilience. In diseased tissues of ginseng, there were more lipid metabolites with significantly altered contents. For example, 
phosphatidylcholine (PE) and phosphatidylethanolamine (PC) are lipids that are present in large amounts in cell membranes. An increase in the PC/PE ratio has been reported to be a marker of the membrane bilayer's structural stability to prevent membrane degradation [57]. Interestingly, we detected a significant increase in several PC levels and a substantial decrease in PE levels in diseased tissues of ginseng. Therefore, diseased tissues may have a stronger tendency to maintain biofilm integrity and stability than healthy tissues.

The accumulation of alkaloids is a vital defence strategy for plants in response to biotic stresses, and alkaloids are a crucial class of compounds involved in plant chemical defences [58]. In diseased tissues, we found an upregulation of the accumulation of numerous alkaloid metabolites. The KEGG enrichment results of differentially accumulated metabolites also contained numerous alkaloid biosynthetic pathways (Fig. 2c). This result suggests that alkaloid metabolites are essential for the stress response of ginseng. Chemical defences against external stress involving alkaloids may occur in diseased tissues [55].

\section{Conclusions}

The current study performed a comparative metabolome and transcriptome analysis of GRS and HG tissues. An analysis of the pathways associated with the plant resistance response revealed that hormone signalling pathways, lignin biosynthesis, ROS regulation by peroxisomes changes during the plant-pathogen response. Additionally, the diseased tissues' metabolic patterns changed, and the production and accumulation of many secondary metabolites may play an essential role in GRS. Finally, a model was proposed to explain the response of ginseng to GRS (Fig. 8). Our results provide new insights into ginseng's response to GRS, revealing the potential molecular mechanisms of this disease in ginseng.

\section{Methods}

\section{Plant material and tissue collection}

All ginseng samples (5 years old) were collected from the same ginseng farm in Hunchun city, Jilin Province, China $\left(42.86^{\prime} \mathrm{N}\right.$ and $\left.130.37^{\prime} \mathrm{E}\right)$. In the previous survey, this was a ginseng farm with a high rusty root index [59].

GRS diseased tissues (main root) and HG healthy tissues were collected as samples frozen in liquid nitrogen immediately and stored at $-80^{\circ} \mathrm{C}$ before use. Independent biological replicates were prepared, and each replicate included root materials from three or more ginseng plants.

\section{RNA extraction, library preparation, clustering and sequencing}

Six complementary DNA (cDNA) libraries, three for GRS tissues, and three for HG tissues, were constructed. TRIzol reagent (Invitrogen, Carlsbad, CA) was used to isolate each sample's total RNA. RNA degradation and contamination were monitored on $1 \%$ agarose gels. RNA purity was checked using a NanoPhotometer ${ }^{\circ}$ spectrophotometer (IMPLEN, CA, USA). RNA concentration was measured using Qubit ${ }^{\circ}$ RA Assay Kit in a Qubit $^{\circ}$ 2.0 Fluorometer (Life Technologies, CA, USA). RNA integrity was assessed using an RNA Nano 6000 Assay Kit and the Bioanalyzer 2100 system (Agilent Technologies, CA, USA).

A total amount of $3 \mu \mathrm{g}$ of RNA per sample was used as the input material for RNA sample preparation. Sequencing libraries were generated using an NEBNext ${ }^{\circ}$ Ultra $^{\text {Tn }}$ RNA Library Prep Kit for Illumina ${ }^{\circ}$ (NEB, USA), following the manufacturer's recommendations, and index codes were added to attribute sequences to each sample. Briefly, mRNA was purified from the total RNA using poly-T oligo-attached magnetic beads. Fragmentation was conducted using divalent cations under elevated temperatures in NEBNext First Strand Synthesis Reaction Buffer (5X). First strand cDNA was synthesized using a random hexamer primer and M-MuLV Reverse Transcriptase (RNase H-). Second strand cDNA synthesis was subsequently performed using DNA Polymerase $\mathrm{I}$ and RNase $\mathrm{H}$. The remaining overhangs were converted into blunt ends via exonuclease/polymerase activities. After adenylation of the 3' ends of DNA fragments, the NEBNext adaptor with a hairpin loop structure was ligated to prepare for hybridization. To preferentially select cDNA fragments that were $150-200 \mathrm{bp}$ in length, the library fragments were purified with the AMPure XP system (Beckman Coulter, Beverly, USA). Then, $3 \mu \mathrm{l}$ of USER Enzyme (NEB, USA) was used with size-selected, adaptor-ligated cDNA at $37^{\circ} \mathrm{C}$ for $15 \mathrm{~min}$ followed by 5 min at $95^{\circ} \mathrm{C}$ before PCR. PCR was performed with Phusion High-Fidelity DNA polymerase, universal PCR primers, and an index (X) primer. PCR products were purified (AMPure XP system), and library quality was assessed on the Agilent Bioanalyzer 2100 system.

After cluster generation, RNA library preparations were sequenced on an Illumina HiSeq PE150 platform, and $150 \mathrm{bp}$ paired-end reads were generated. The raw RNA-seq data are freely available in the NCBI database under accession no. PRJNA684799.

\section{Transcriptome data analysis}

Raw data (raw reads) in fastq format were first processed through in-house Perl scripts. A certain length range was then chosen from clean reads to perform all of the downstream analyses.

The reference genome and gene model annotation files were downloaded from the genome website directly [60]. The reference genome index was built using STAR, and paired-end clean reads were aligned to the reference genome using STAR (v.2.5.1b). STAR used the maximal 
mappable prefix (MMP) method to generate a precise mapping result for junction reads. HTSeq (v.0.6.0) was used to count the number of reads mapped to each gene. The expected number of fragments per kilobase of transcripts per million mapped reads (FPKM) of each gene was calculated based on the length and mapped read count for that gene.

Differential expression analysis of the two groups was performed using the DESeq2 $\mathrm{R}$ package (v.1.10.1). The resulting $P$ values were adjusted using Benjamini and Hochberg's approach for controlling the false discovery rate.

GO (http://www.geneontology.org/) enrichment analysis of DEGs was implemented by the GOseq-based Wallenius noncentral hypergeometric distribution, in which gene length bias was corrected [61]. In addition, we performed KEGG enrichment analysis on DEGs using KOBAS 2.0 software [62].

\section{Metabolome analysis}

First, we extracted the metabolites. Tissues (100 mg) were individually ground with liquid nitrogen, and the homogenate was resuspended in prechilled $80 \%$ methanol and $0.1 \%$ formic acid by vortexing. The samples were incubated on ice for $5 \mathrm{~min}$ and then were centrifuged at $15,000 \mathrm{rpm}$ and $4{ }^{\circ} \mathrm{C}$ for $5 \mathrm{~min}$. Some supernatants were diluted with UHPLC-MS/MS grade water to a final concentration of $53 \%$ methanol. The samples were subsequently transferred to a fresh Eppendorf tube and then centrifuged at $15000 \times \mathrm{g}$ and $4{ }^{\circ} \mathrm{C}$ for $10 \mathrm{~min}$.

UHPLC-MS/MS analysis was then performed using a Vanquish UHPLC system (Thermo Fisher, Germany) coupled with an Orbitrap Q Exactive ${ }^{\text {tm }}$ HF-X mass spectrometer (Thermo Fisher, Germany). Samples were injected onto a Hypesil Gold column $(100 \times 2.1 \mathrm{~mm}$, $1.9 \mu \mathrm{m})$ using a 17 -min linear gradient at a flow rate of $0.2 \mathrm{~mL} / \mathrm{min}$. The eluents for the positive polarity mode were eluent A $(0.1 \%$ FA in water) and eluent B (methanol). The eluents for the negative polarity mode were eluent $\mathrm{A}(5 \mathrm{mM}$ ammonium acetate, $\mathrm{pH} 9.0)$ and eluent $\mathrm{B}$ (methanol). The solvent gradient was set as follows: $2 \%$ B, 1.5 min; $2-100 \%$ B, $12.0 \mathrm{~min} ; 100 \%$ B, $14.0 \mathrm{~min}$; $100-2 \%$ B, 14.1 min; 2\% B, 17 min. A Q Exactive ${ }^{\mathrm{me}} \mathrm{HF}-\mathrm{X}$ mass spectrometer was operated in positive/negative polarity mode with a spray voltage of $3.2 \mathrm{kV}$, capillary temperature of $320^{\circ} \mathrm{C}$, sheath gas flow rate of $40 \mathrm{arb}$, and aux gas flow rate of 10 arb.

The raw data files generated by UHPLC-MS/MS were processed using the Compound Discoverer 3.1 (CD3.1, Thermo Fisher) to perform peak alignment, peak selection, and quantitation for each metabolite. The main parameters were set as follows: retention time tolerance, $0.2 \mathrm{~min}$; actual mass tolerance, $5 \mathrm{ppm}$; signal intensity tolerance, $30 \%$; signal/noise ratio, 3; and minimum intensity, 100,000. After that, peak intensities were normalized to the total spectral intensity. The normalized data were used to predict the molecular formula based on additive ions, molecular ion peaks, and fragment ions. Then, peaks were matched with the mzCloud, mzVault, and MassList databases to obtain accurate qualitative and relative quantitative results.

PCA and PLS-DA were performed using SIMCA-P 14.1 (Umetrics, Umea, Sweden). The metabolites with a VIP $>1, P$-value $<0.05$ and fold change $>2$ were considered to be differential metabolites. KEGG enrichment analysis of differentially accumulated metabolites was performed using KOBAS 2.0 software [62].

\section{Combined analysis of DEGs and differentially accumulated metabolites}

The obtained differentially accumulated metabolites and DEGs were mapped to KEGG pathway for integration. In addition, we performed correlation analysis on transcriptomic and metabolomic data to explore correlations between DEGs and metabolites. The PCC of DEGs and differentially accumulated metabolites was calculated using the COR function in R. The genes and metabolites with a PCC $>0.8$ in the KEGG pathway were used to establish the related network that was visualized by Cytoscape software.

\section{Quantitative real-time qPCR}

Quantitative PCR was performed on a Real-Time PCR System (Lightcycler 96, Roche, Switzerland) using $2 \times$ RealStar Green Fast Mixture (GenStar, China). For each reaction, $0.5 \mu \mathrm{l}$ of the forward and reverse primers and $2 \mu \mathrm{l}$ of the cDNA template were added. All of the primers used in this study are listed in Table S7. The relative gene expression level was calculated according to the $2^{-\Delta \Delta \mathrm{Ct}}$ method [63].

\section{Statistical analysis}

Statistical analysis was performed with SPSS 20.0 software. All of the data were expressed as the mean \pm SEM. $P<0.05$ was considered statistically significant. RT-PCR results were analysed using a Student's $t$-test. Significance was established at $P<0.05$.

\section{Abbreviations}

GRS: Ginseng rusty root symptoms; HG: Healthy ginseng; UHPLC-MS/ MS: Liquid chromatography-tandem mass spectrometry; KEGG: Kyoto Encyclopedia of Genes and Genomes; DEGs: Differentially expressed genes; GO: Gene Ontology; BP: Biological process; CC: Cellular component; MF: Molecular function; PCA: Principal component analysis; OPLS-DA: Partial least-squares discriminant analysis; MeJA: Methyl jasmonate; ABA: Abscisic acid; SA: Salicylic acid; PP2Cs: Protein phosphatases type-2C; SnRK: SNF1Related Protein Kinase; COI1: Coronatine insensitive 1; JAZ: Jasmonate-zim domain; NPR1: Protein non-expressor pr gene 1; PAL: Phenylalanine ammonia-lyase; OMT1: O-methyltransferase1; 4CL: 4-coumarate: coenzyme A ligase; CCR: Cinnamoyl coenzyme A reductase; ROS: Reactive oxygen species; CAT: Catalase; SOD: Superoxide dismutase; RBOHs: Respiratory burst oxidase 
homologues; FLS2: Flagellin sensing 2; PAMP: Pathogen-associated molecular patterns; PTI: PAMP-triggered immunity; ETI: Effector-triggered immunity; PPC: Pearson correlation coefficient; qPCR: Quantitative polymerase chain reaction; CDNA: Complementary DNA; MMP: Maximal mappable prefix; FPKM: Fragments per kilobase of transcript per million mapped reads

\section{Supplementary Information}

The online version contains supplementary material available at https://doi. org/10.1186/s12870-021-03001-w.

Additional file 1: Table S1. Summary of RNA-seq data and mapped reads for mRNA.

Additional file 2: Table S2. Gene Ontology results of all the enriched terms for the upregulated mRNAs in GRS tissues.

Additional file 3: Table S3. Gene Ontology results of all the enriched terms for the downregulated mRNAs in GRS tissues.

Additional file 4: Table S4. All pathways associated with DEGs based on KEGG enrichment analysis.

Additional file 5: Table S5. Results of UHPLC-MS/MS analysis in positive ion mode.

Additional file 6: Table S6. Results of UHPLC-MS/MS analysis in negative ion mode.

Additional file 7: Table S7. Primer sequences of the genes for $\mathrm{QPCR}$ verification.

Additional file 8: Figure S1. Plant hormone contents and heatmaps of DEGs.

Additional file 9: Figure S2. The plant hormone signal transduction pathway.

Additional file 10: Figure S3. Heatmap of DEGs related to peroxisome synthesis.

Additional file 11: Figure S4. The peroxisome pathway.

Additional file 12: Figure S5. The plant-pathogen interaction pathway. Additional file 13: Figure S6. Heatmap of DEGs related to plantpathogen interaction.

\section{Acknowledgments}

Not applicable.

\section{Authors' contributions}

L.Z. designed the experiment; X.B. and S.X. analyzed the samples; Y.Z., H.Y. and Y.H. analyzed the data; X.B. wrote the first draft of the manuscript. All authors have read and approved the final mauscript.

\section{Funding}

This study was supported in part by grants from the National key R\&D program (grant No. 2017YFC1702100) and the Jilin Province Major Science and Technology Special Project (grant No. 20200504003YY). Funders helped design the experiment for this study.

\section{Availability of data and materials}

All relevant supporting data sets are included in the article and its supplemental files.

\section{Declarations}

\section{Ethics approval and consent to participate}

Not applicable.

\section{Consent for publication}

Not applicable.

\section{Competing interests}

The authors declare that they have no competing interests.

\section{Author details}

'College of Chinese Medicinal Materials, Jilin Agricultural University, Changchun 130118, Jilin Province, China. ${ }^{2}$ State Local Joint Engineering Research Center of Ginseng Breeding and Application, Changchun, China. ${ }^{3}$ Jilin Provincial Ginseng and Pilose Antler Office, Changchun, China.

Received: 20 February 2021 Accepted: 27 April 2021

Published online: 13 May 2021

\section{References}

1. Proctor JTA, Bailey WG. Ginseng: industry, botany, and culture. Hortic Rev. 1987:9:187-236.

2. Zhou Y, Yang Z, Gao L, Liu W, Liu R, Zhao J, et al. Changes in element accumulation, phenolic metabolism, and antioxidative enzyme activities in the red-skin roots of Panax ginseng. J Ginseng Res. 2016;41(3):307-15. https://doi.org/10.1016/j.jgr.2016.06.001.

3. Wang Q, Sun H, Xu C, Ma L, Li M, Shao C, et al. Analysis of rhizosphere bacterial and fungal communities associated with rusty root disease of Panax ginseng. Appl Soil Ecol. 2019;138:245-52. https://doi.org/10.1016/j.a psoil.2019.03.012.

4. Rahman M, Punja ZK. Biochemistry of ginseng root tissues affected by rusty root symptoms. Plant Physiol Biochem. 2005;43(12):1103-14. https://doi. org/10.1016/j.plaphy.2005.09.004

5. Reeleder RD, Hoke SMT, Zhang Y. Rusted root of ginseng (Panax quinquefolius) is caused by a species of Rhexocercosporidium. Phytopathology. 2006;96(11):1243-54. https://doi.org/10.1094/PHYTO96-1243.

6. Lu XH, Zhang XM, Jiao XL, Hao JJ, Zhang XS, Luo Y, et al. Taxonomy of fungal complex causing red-skin root of Panax ginseng in China. J Ginseng Res. 2020;44(3):506-18. https://doi.org/10.1016/j.jgr.2019.01.006.

7. Farh ME, Kim YJ, Kim YJ, Yang DC. Cylindrocarpon destructans/llyonectria radicicola-species complex: causative agent of ginseng root-rot disease and rusty symptoms. J Ginseng Res. 2018;42(1):9-15. https://doi.org/10.1016/j. jgr.2017.01.004.

8. Liu D, Sun H, Ma H. Deciphering microbiome related to rusty roots of Panax ginseng and evaluation of antagonists against pathogenic Ilyonectria. Front Microbiol. 2019;10:1350. https://doi.org/10.3389/fmicb.2019.01350.

9. Wang Z, Gerstein M, Snyder MJNRG. RNA-Seq: a revolutionary tool for transcriptomics. Nat Rev Genet. 2009;10(1):57-63. https://doi.org/10.1038/ nrg2484.

10. Sun L, Wang J, Song K, Sun Y, Qin Q, Xue YJSR. Transcriptome analysis of rice (Oryza sativa L.) shoots responsive to cadmium stress. Sci Rep. 2019;9(1): $1-10$.

11. Zhang Y, Gao X, Li J, Gong X, Yang P, Gao J, et al. Comparative analysis of proso millet ( Panicum miliaceum L.) leaf transcriptomes for insight into drought tolerance mechanisms. BMC Plant Biol. 2019;19(1):1-17.

12. Li H, Mo Y, Cui Q, Yang X, Guo Y, Wei C, et al. Transcriptomic and physiological analyses reveal drought adaptation strategies in droughttolerant and -susceptible watermelon genotypes. Plant Sci. 2019;278:32-43. https://doi.org/10.1016/j.plantsci.2018.10.016.

13. Kang X, Wang L, Guo Y, Arifeen MZU, Cai X, Xue Y, et al. A comparative Transcriptomic and proteomic analysis of Hexaploid Wheat's responses to colonization by bacillus velezensis and Gaeumannomyces graminis, both separately and combined. Mol Plant Microbe. 2019;32(10):1336-47. https:// doi.org/10.1094/MPMI-03-19-0066-R.

14. Bano A, Muqarab RJPB. Plant defence induced by PGPR against Spodoptera litura in tomato (Solanum lycopersicum L.). Plant Biol. 2017;19(3):406-12. https://doi.org/10.1111/plb.12535.

15. Guo J, Wu Y, Wang G, Wang T, Cao F. Integrated analysis of the transcriptome and metabolome in young and mature leaves of Ginkgo biloba L. Ind Crop Prod. 2020;143:111906. https://doi.org/10.1016/j.indcrop.2 019.111906.

16. Cevallos-Cevallos JM, García-Torres R, Etxeberria E, Reyes-De-Corcuera J. GCMS analysis of headspace and liquid extracts for Metabolomic differentiation of citrus Huanglongbing and zinc deficiency in leaves of 'Valencia' sweet Orange from commercial groves. Phytochem Anal. 2011; 22(3):236-46. https://doi.org/10.1002/pca.1271.

17. Hamzehzarghani H, Paranidharan V, Abu-Nada Y, Kushalappa AC, Mamer O, Somers D. Metabolic profiling to discriminate wheat near isogenic lines, with quantitative trait loci at chromosome $2 \mathrm{DL}$, varying in resistance to fusarium head blight. Can J Plant Sci. 2008;88(4):789-97. 
18. Barding GA, Béni S, Fukao T, Bailey-Serres J, Larive CK. Comparison of GC-MS and NMR for metabolite profiling of Rice subjected to submergence stress. Proteome Res. 2013;12(2):898-909. https://doi.org/10.1021/pr300953k.

19. Jahangir M, Abdel-Farid IB, Choi YH, Verpoorte R. Metal ion-inducing metabolite accumulation in Brassica rapa. J Plant Physiol. 2008;165(14):142937. https://doi.org/10.1016/j.jplph.2008.04.011.

20. Jayakodi M, Lee S, Lee YS, Park H, Kim N, Jang W, et al. Comprehensive analysis of Panax ginseng root transcriptomes. BMC Plant Biol. 2015;15(1):1-12.

21. Jones JD, Dangl JL. The plant immune system. Nature. 2006:444(7117):3239. https://doi.org/10.1038/nature05286.

22. Bari $\mathrm{R}$, Jones JD. Role of plant hormones in plant defence responses. Plant Mol Biol. 2009;69(4):473-88. https://doi.org/10.1007/s11103-008-9435-0.

23. Fujii H, Chinnusamy V, Rodrigues A, Rubio S, Antoni R, Park SY, et al. In vitro reconstitution of an abscisic acid signalling pathway. Nature. 2009;462(7273): 660-4. https://doi.org/10.1038/nature08599.

24. Chini A, Fonseca S, Fernandez G, Adie B, Chico J, Lorenzo O, et al. The JAZ family of repressors is the missing link in jasmonate signalling. Nature. 2007; 448(7154):666-71. https://doi.org/10.1038/nature06006.

25. Chico JM, Chini A, Fonseca S, Solano R. JAZ repressors set the rhythm in jasmonate signaling. Curr Opin Plant Biol. 2008;11(5):486-94. https://doi. org/10.1016/j.pbi.2008.06.003.

26. Canet JV, Dobon A, Roig A, Tornero PJPC. Environment. Structure-function analysis of npr1 alleles in Arabidopsis reveals a role for its paralogs in the perception of salicylic acid. Plant Cell Environ. 2010;33(11):1911-22. https:// doi.org/10.1111/j.1365-3040.2010.02194.x.

27. Johnson C, Boden E, Arias JJTPC. Salicylic acid and NPR1 induce the recruitment of trans- activating TGA factors to a defense gene promoter in Arabidopsis. Plant Cell. 2003;15(8):1846-58. https://doi.org/10.1105/tpc. 012211.

28. Irieda H, Inoue Y, Mori M, Yamada K, Oshikawa Y, Saitoh H, et al. Conserved fungal effector suppresses PAMP-triggered immunity by targeting plant immune kinases. P Natl A Sci. 2019;116(2):496-505. https://doi.org/10.1073/ pnas.1807297116.

29. Marino D, Dunand C, Puppo A. Pauly NJTiPS. A burst of plant NADPH oxidases. Trends Plant Sci. 2012;17(1):9-15. https://doi.org/10.1016/j.tplants.2 011.10.001.

30. Zhang J, Shao F, Li Y, Cui H, Chen L, Li H, et al. A pseudomonas syringae effector inactivates MAPKs to suppress PAMP-induced immunity in plants. Cell Host Microbe. 2007;1(3):175-85. https://doi.org/10.1016/j.chom.2007.03.006.

31. Torres MA, Dangl JL, Jones JDG. Arabidopsis gp91phox homologues AtrbohD and AtrbohF are required for accumulation of reactive oxygen intermediates in the plant defense response. P Natl A Sci. 2002;99(1):517-22. https://doi.org/10.1073/pnas.012452499.

32. Robatzek S, Chinchilla D, Boller TJG. Development. Ligand-induced endocytosis of the pattern recognition receptor FLS2 in Arabidopsis. Genes Dev. 2006;20(5):537-42. https://doi.org/10.1101/gad.366506.

33. Zheng Z, Qamar SA, Chen Z, Mengiste TJPJ. Arabidopsis WRKY33 transcription factor is required for resistance to necrotrophic fungal pathogens. Plant J. 2006;48(4):592-605. https://doi.org/10.1111/j.1365-313X.2 006.02901.x

34. Liu J, Elmore JM, Coaker GJPS. Behavior. Investigating the functions of the RIN4 protein complex during plant innate immune responses. Plant Signal Behav. 2009;4(12):1107-10. https://doi.org/10.4161/psb.4.12.9944.

35. Takahashi A, Casais C, Ichimura K, Shirasu K. HSP90 interacts with RAR1 and SGT1 and is essential for RPS2-mediated disease resistance in Arabidopsis. P Natl A Sci. 2003;100(20):11777-82. https://doi.org/10.1073/pnas.2033934100.

36. Azevedo C, Betsuyaku S, Peart JR, Takahashi A, Noel LD, Sadanandom A, et al. Role of SGT1 in resistance protein accumulation in plant immunity. EMBO J. 2006;25(9):2007-16. https://doi.org/10.1038/sj.emboj.7601084.

37. Kang L, Li J, Zhao T, Xiao F, Tang X, Thilmony R, et al. Interplay of the Arabidopsis nonhost resistance gene $\mathrm{NHO} 1$ with bacterial virulence. P Natl A Sci. 2003;100(6):3519-24

38. Czarnocka W, Karpinski S. Friend or foe? Reactive oxygen species production, scavenging and signaling in plant response to environmental stresses. Free Radical Bio Med. 2018;122:4-20. https://doi.org/10.1016/j.freera dbiomed.2018.01.011.

39. Gill SS, Tuteja N. Reactive oxygen species and antioxidant machinery in abiotic stress tolerance in crop plants. Plant Physiol Biochem. 2010;48(12): 909-30. https://doi.org/10.1016/j.plaphy.2010.08.016.
40. Corpas FJ, Barroso JB, Rio LA. Peroxisomes as a source of reactive oxygen species and nitric oxide signal molecules in plant cells. Trends Plant Sci. 2001;6(4):145-50. https://doi.org/10.1016/S1360-1385(01)01898-2.

41. Lismont C, Koster J, Provost S, Baes M, Van Veldhoven PP, Waterham HR, et al. Deciphering the potential involvement of PXMP2 and PEX11B in hydrogen peroxide permeation across the peroxisomal membrane reveals a role for PEX11B in protein sorting. Biochim Biophys Acta Biomembr. 2019; 1861(10):182991. https://doi.org/10.1016/j.bbamem.2019.05.013.

42. Lee MY, Sumpter R Jr, Zou Z, Sirasanagandla S, Wei Y, Mishra P, et al. Peroxisomal protein PEX13 functions in selective autophagy. EMBO Rep. 2017;18(1):48-60. https://doi.org/10.15252/embr.201642443.

43. Cross LL, Ebeed HT, Baker A. Peroxisome biogenesis, protein targeting mechanisms and PEX gene functions in plants. Biochim Biophys Acta. 2016; 1863(5):850-62. https://doi.org/10.1016/j.bbamcr.2015.09.027.

44. Mittler R. Oxidative stress, antioxidants and stress tolerance. Trends Plant Sci. 2002;7(9):405-10. https://doi.org/10.1016/S1360-1385(02)02312-9.

45. Mwamba T, Islam F, Ali B, Lwalaba J, Gill R, Zhang F, et al. Comparative metabolomic responses of low-and high-cadmium accumulating genotypes reveal the cadmium adaptive mechanism in Brassica napus. Chemosphere. 2020;250:126308. https://doi.org/10.1016/j.chemosphere.2 020.126308 .

46. Chen J, Ullah C, Reichelt M, Gershenzon J, Hammerbacher A. Sclerotinia sclerotiorum circumvents flavonoid defenses by catabolizing Flavonol glycosides and Aglycones. Plant Physiol. 2019;180(4):1975-87. https://doi. org/10.1104/pp.19.00461

47. Van den Ende W, Valluru R. Sucrose, sucrosyl oligosaccharides, and oxidative stress: scavenging and salvaging? J Exp Bot. 2008;60(1):9-18. https://doi. org/10.1093/jxb/ern297.

48. KEUNEN E, PESHEV D, VANGRONSVELD J, VAN DEN ENDE W, CUYPERS A Plant sugars are crucial players in the oxidative challenge during abiotic stress: extending the traditional concept. Plant Cell Environ. 2013;36(7): 1242-55. https://doi.org/10.1111/pce.12061.

49. Moura JCMS, Bonine CAV, Viana JDOF, et al. Abiotic and biotic stresses and changes in the lignin content and composition in plants. J Integr Plant Biol. 2010;52(4):360-76. https://doi.org/10.1111/j.1744-7909.2010.00892.x.

50. Rogers LA, Campbell MM. The genetic control of lignin deposition during plant growth and development. New Phytol. 2004;164(1):17-30. https://doi. org/10.1111/j.1469-8137.2004.01143.x.

51. Rastogi S, Dwivedi UN. Manipulation of lignin in plants with special reference to O-methyltransferase. PInat Sci. 2008;174(3):264-77. https://doi. org/10.1016/j.plantsci.2007.11.014

52. Lauvergeat V, Lacomme C, Lacombe E, Lasserre E, Roby D, Grima-Pettenati J. Two cinnamoyl-CoA reductase (CCR) genes from Arabidopsis thaliana are differentially expressed during development and in response to infection with pathogenic bacteria. Phytochemistry. 2001;57(7):1187-95. https://doi. org/10.1016/S0031-9422(01)00053-X.

53. Anterola AM, Lewis NG. Trends in lignin modification: a comprehensive analysis of the effects of genetic manipulations/mutations on lignification and vascular integrity. Phytochemistry. 2002;61(3):221-94. https://doi.org/1 0.1016/S0031-9422(02)00211-X.

54. Gomaa NH, Hassan MO, Fahmy GM, González L, Hammouda O, Atteya AM. Flavonoid profiling and nodulation of some legumes in response to the allelopathic stress of Sonchus oleraceus L. Acta Bot Bras. 2015;29(4):553-60. https://doi.org/10.1590/0102-33062015abb0153.

55. Zaynab M, Fatima M, Abbas S, Sharif Y, Umair M, Zafar MH, et al. Role of secondary metabolites in plant defense against pathogens. Microb Pathog. 2018;124:198-202. https://doi.org/10.1016/j.micpath.2018.08.034.

56. Gigon A, Matos A-R, Laffray D, et al. Effect of drought stress on lipid metabolism in the leaves of Arabidopsis thaliana (ecotype Columbia). Ann Bot-London. 2004;94(3):345-51. https://doi.org/10.1093/aob/mch150.

57. Toumi I, Gargouri M, Nouairi I, Moschou P, Salem-Fnayou AB, Mliki A, et al. Water stress induced changes in the leaf lipid composition of four grapevine genotypes with different drought tolerance. Biol Plant. 2008;52(1): 161-4. https://doi.org/10.1007/s10535-008-0035-2.

58. Mithöfer A, Maffei ME. General mechanisms of plant defense and plant toxins; 2016. p. 1-22

59. Bian X, Xiao S, Zhao Y, Xu Y, Yang H, Zhang L. Comparative analysis of rhizosphere soil physiochemical characteristics and microbial communities between rusty and healthy ginseng root. Sci Rep. 2020;10(1):15756. https:// doi.org/10.1038/s41598-020-71024-8. 
60. Jayakodi M, Choi B-S, Lee S-C, Kim N-H, Park JY, Jang W, et al. Ginseng genome database: an open-access platform for genomics of Panax ginseng BMC Plant Biol. 2018;18(1):62. https://doi.org/10.1186/s12870-018-1282-9.

61. Young MD, Wakefield MJ, Smyth GK, Oshlack AJGB. Gene ontology analysis for RNA-seq: accounting for selection bias. Genome Biol. 2010;11(2):1-12.

62. Mao X, Cai T, Olyarchuk JG, Wei LB. Automated genome annotation and pathway identification using the KEGG Orthology (KO) as a controlled vocabulary. Bioinformatics. 2005;21 (19):3787-93. https://doi.org/10.1093/ bioinformatics/bti430.

63. Arocho A, Chen B, Ladanyi M, Pan QJDMP. Validation of the 2-DeltaDeltaCt calculation as an alternate method of data analysis for quantitative PCR of BCR-ABL P210 transcripts. Diagn Mol Pathol. 2006;15(1):56-61. https://doi. org/10.1097/00019606-200603000-00009.

\section{Publisher's Note}

Springer Nature remains neutral with regard to jurisdictional claims in published maps and institutional affiliations.

Ready to submit your research? Choose BMC and benefit from:

- fast, convenient online submission

- thorough peer review by experienced researchers in your field

- rapid publication on acceptance

- support for research data, including large and complex data types

- gold Open Access which fosters wider collaboration and increased citations

- maximum visibility for your research: over $100 \mathrm{M}$ website views per year

At BMC, research is always in progress.

Learn more biomedcentral.com/submissions 